Original Article

\title{
Effectiveness of Vestibular Rehabilitation Exercises On Dizziness- Handicap among Patients with Vertigo Attending ENT OPD at A Selected Hospital
}

\section{Thota Malathi' ${ }^{1}$, A. Seethalakshmi ${ }^{2}$, P. Akila ${ }^{3}$}

${ }^{1}$ Lecturer, Smt. Vijaya Luke College of Nursing, Visakhapatnam, Andhra pradesh, ${ }^{2}$ Reader, Sri Ramachandra University, Porur, Chennai, India, ${ }^{3}$ Professor, Department of Medical surgical Nursing, College of Nursing, Kovai Medical Center Research and Educational trust.

*Corresponding Author : A. Seethalakshmi, Reader, Faculty of Nursing, Sri Ramachandra University, Porur, Chennai, India.

E-mail : seethalakshmi.a@sriramachandra.edu.in

Received

: 13.06 .2016

Review Completed : 30.08.2016

Accepted

$: 29.10 .2016$

Keywords : vestibular rehabilitation, dizziness-handicap, vertigo.

\begin{tabular}{|c|}
\hline Access this article online \\
\hline Quick Response Code \\
\hline
\end{tabular}

\author{
Abstract \\ Objective: The current research aims to identify the effect of Vestibular rehabilitation exercises \\ on the dizziness-handicap among patients with vertigo.
}

Methods: The research design adopted is a pretest-posttest design. 30 patients with vertigo attending the ENT out Patient Department (OPD) who fulfilled the inclusion criteria were selected randomly by lottery method, 15 were assigned to the study and 15 to the control group respectively. Vestibular rehabilitation exercises which include eye, head, sitting, standing $\&$ walking exercises were demonstrated to the study group patients on $1^{\text {st }}$ consultation using a booklet for 20 minutes along with return demonstration. The patients were reinforced to perform the exercises twice daily for 7 days following which the posttest was carried out on the $7^{\text {th }}$ day. The control group received routine care.

Results : The results showed that in the study group $86.7 \%$ had severe level of dizzinesshandicap during the pretest which was reduced after practicing vestibular rehabilitation to $13.3 \%$ during the posttest, whereas in the control group it was $66.7 \%$ and $73.3 \%$ who had severe level of dizziness-handicap during the pretest and the posttest respectively. The mean for dizziness handicap for the study group at pretest was $66.3+14.03$ and at posttest it was 31.07 \pm 18.94 . The control group showed that at pretest the mean was $61.33 \pm 15.24$ and at posttest it was $63.87 \pm 22.35$. This was found to be significant at $p=0.0005$.

Conclusion : The study supported the hypothesis that vestibular rehabilitation exercises had an impact on the dizziness handicap.

\section{Introduction}

Dizziness and vertigo are common medical issues and it affects approximately $20 \%-30 \%$ of the general population. Vertigo can occur in people of all ages. The prevalence of vertigo rises with age and is about 2 to 3 times higher in women than in men. It accounts for about $2-3 \%$ of emergency department visits. The main causes of vertigo are benign paroxysmal positional vertigo, Meniere's disease, vestibular neuritis and labyrinthitis, but may also be caused by a concussion, a vestibular migraine or vertiginous epilepsy. Excessive drinking of alcohol can also cause symptoms of vertigo. ${ }^{1}$ the common problems encountered among vertigo patients were postural instability and disequilibrium which manifested as a result of the vestibular dysfunction. Vestibular dysfunction also increases the risk of falling. Among 546 patients presenting with no known cause of falls to an Accident and Emergency Department in the United Kingdom, 80\% had symptoms of vestibular impairment. People who were 40 years of age and older with symptoms of vestibular dysfunction had a 12 fold increase in the risk of falling. Falls can result in serious consequences to persons' health and quality of life (QOL), especially in older adults. Dizziness, vertigo, and imbalance have disabling effects on people with vestibular disorders; they interrupt their normal life by impairing their performance of daily living activities and their participation 
with the community. ${ }^{2,13}$

The overall incidence of vertigo is 5-10\%, and has reached to $40 \%$ in patients who are older than 40 years of age. In people less than 80 years the prevalence is $17.6 \%$ and in people older than 80 years the prevalence is $31 \%$. A large population survey in Germany determined that the lifetime prevalence of vestibular vertigo in adults aged 18-79 years were $7.8 \%$, the 1 -year prevalence was $5.2 \%$, and the incidence was $1.5 \%{ }^{3,11}$ Dizziness and vertigo rank among the most common complaints reported in medicine in the United States and around the world. Approximately $20 \%$ to $35 \%$ of individuals in the general population report dizziness episodes to a physician or via a national health survey. In the United States, about 7.5 million patients with dizziness were examined in ambulatory care settings between 1999 and 2000. From 2001 to 2004, over 69 million Americans over the age of 40 reported vestibular dysfunction. ${ }^{4}$

The prevalence rate of vertigo in the United States was about $2.5 \%$ among 8 million patients. ${ }^{5,20}$ The impact of dizziness in the US population identified by the US National Health Interview Surveys in 1986 among 3, 00,000 people found that $26 \%$ of patients with vertigo were unable to work. ${ }^{6}$

In India, majority of the people with vertigo were in the third and fourth decade, only $11 \%$ in sixth and eighth decade. Raman Abrol et al. (2011), in a study on prevalence of vertigo in adult population, among $71(45 \%)$ vertigo patients 31 were attributed to cardiovascular disease which was found to increase with age. It was common in the $6^{\text {th }}$ decade, with the females being 4 times more affected than the males. Vertigo was associated with hypertension (32.4\%) followed by postural hypotension (5.6\%). Anemia wasseen in $4.2 \%$ of the cases. ${ }^{7}$

$M$ any vestibular deficits are seen in vertigo which affects the activities of daily living among individuals. These vestibular deficits manifest as dizziness, vertigo, and imbalance. In Europe, dizziness and vertigo are also the frequent complaints in emergency settings. About $15 \%$ of the world's population has dizziness. Of these, $40 \%$ have otologic dizziness, $10 \%$ have central dizziness, $25 \%$ have medical dizziness and $25 \%$ have undiagnosed dizziness. ${ }^{8}$ Patients with vertigo commonly experience difficulties such as nausea or vomiting, unsteadiness, postural instability, falls, thought disturbances and difficulty in speaking, a lowered level of consciousness and hearing loss may also occur. ${ }^{9}$

Vestibular disorders have debilitating consequences both in terms of physical and psychological domains that impair individual's activities of daily living (ADL) and health related quality of life (QOL).Unsteadiness, imbalance, and falls are all physical sequelae of vestibular disorders that contributes to individual's disability. Psychological disturbances including panic disorders, agoraphobia, anxiety, and major depression were reported by approximately $50 \%$ of individuals with vestibular disorders. 10

People with vestibular disorders avoid a wide range of activities due to the nature of their condition. The vestibular disorders are also detrimental due to the fear of provoking symptoms. Such avoidance affects the individual's ability in adaptation, since controlled exposure is necessary for adaptation, and contributes to greater disability. Therefore, many basic and essential activities become unsafe and difficult to perform such as negotiating stairs, climbing ladders, driving, and shopping because of fear and avoidance behavior. Gradually, persons with vestibular disorders may become limited in essential daily living activities and restrict in participating in the community.

Activities and participation make up the second component of the functioning and disability part of the International Classification of Functioning, Disability and Health (ICF). Activities are defined as the execution of a task or action by an individual and participation is defined as involvement in a life situation. The negative aspects of activities and participation are called activity limitations and participation restrictions. This includes the difficulty an individual faces in executing activities or has problems in 
managing life situations. The ICF framework has not distinguished between activities and participation in the current version of the classification system; therefore, activities and participation have shared domains. Many instruments have been developed to measure the extent of activity limitations based on the ICF in different populations. However, there is no outcome measure that assesses the extent of activity limitations and/or participation restrictions in individuals with vestibular disorders. ${ }^{12}$

The problems faced by the patients with vertigo have an effect on the physical, psychological and social domains and also limits the performance of their activities of daily living. In addition to the negative impact on the patient from a humanistic perspective, vertigo has considerable impact on work productivity and healthcare resource use. Many research studies showed that the vestibular rehabilitation exercises have an impact in the treatment of vertigo. Vestibular rehabilitation exercises had been popularized from the $19^{\text {th }}$ century and have been found effective in the treatment of vertigo. ${ }^{14}$ In 143 primary care patients with dizziness and vertigo, vestibular rehabilitation exercises improved nystagmus, postural control, movement-provoked dizziness, and subjective indexes of symptoms and distress. ${ }^{15}$

Vertigo exercises help the particles in the semicircular canals that do not cause vertigo. These exercises stop vertigo for some months or even years, the condition may still return hence it is necessary to motivate the patients to perform the vertigo exercises regularly so that they can have a better quality of life.

Vertigo management- Global scenario: The assessment of the burden and impact of vertigo among 4,294 patients from 13 countries of which 4,105 patients participated, revealed that half were employed. Among the employed population, $69.8 \%$ had reduced their workload, $63.3 \%$ had lost their working days, $4.6 \%$ had changed their jobs and $5.7 \%$ had given up their jobs, due to vertigo symptoms. The healthcare services utilization was also high. ${ }^{16}$
Vertigo management literature search in India identified that the assessment of clinical and cost effectiveness of booklet based vestibular rehabilitation in primary care for which the vertigo patients received booklet based vestibular rehabilitation and telephonic reminders to enhance the practice of vestibular rehabilitation exercises at home daily for up to 12 weeks along with cognitive behavioral techniques to promote positive beliefs and treatment adherence revealed that vertigo had improved among 12 patients within the duration of one year. ${ }^{17}$

The nursing care prevailing with regards to vertigo is to limit foods high in salt and sugar. Patients are encouraged to eat meals at regular intervals, to stay hydrated and drink plenty of fluids daily, include fresh fruits and vegetables in the diet and limit alcohol intake as they may change the volume and concentration of the inner ear fluid and may worsen the condition. The nurse should teach and motivate the patients to practice vestibular rehabilitation exercises regularly. ${ }^{18}$

In an epidemiologic analysis of the elderly (both men and women) aged 60 years and above living in a community it was found that $19-29 \%$ of all women and $3-14 \%$ of all men complained of dizziness. ${ }^{19}$

Postural disturbances in a population of 425 women and 333 men aged 65 years and above concluded that $40 \%$ of women and $30 \%$ of men reported disturbances in balance, related to concepts such as unsteadiness, feeling of rotation, impending fainting, dizziness and vertigo. Postural disturbances had been present for more than 6 months in $85 \%$, one third of the population had daily or constant troubles and one tenth of them had these problemsoccasionally.

The effectiveness of vestibular rehabilitation therapy in patients suffering with BPPV among 15 patients with BPPV who were selected in the outpatient department of Sai Sanjeevani Clinic, Mumbai for a period of 3 weeks. The study showed that the vestibular rehabilitation was a powerful method for functional restoration of a patient with BPPV. It also helped the patients to perform their day- 
to-day activities and reduces vertigo-phobia and alleviate symptoms of vertigo. It was also found to improve the balance. $^{22}$

Identification of the indications, mechanisms and key exercises for vestibular rehabilitation therapyfor 60 patients who were selected randomly, showed that the vestibular rehabilitation therapy could be given for any patient regardless of age and the recovery could be facilitated as long as the exercises were performed several timesa day. ${ }^{23}$

A study to investigate whether vestibular rehabilitation could improve balance, reduce self-perceived handicap because of dizziness and if possible reduce falls among elderly people in a primary health care centre revealed that among 58 patients aged 65 years and above the vestibular rehabilitation improved balance among elderly with multisensory dizziness and also vertigo was found to be a common cause of falls in these patients. Vestibular rehabilitation was found to be a feasible treatment modality. ${ }^{24}$

The impact of vestibular rehabilitation exercises can be determined by the level of reduction in the dizziness handicap which is the aim of the current study.

\section{Methodology}

\section{Study area and period}

The study was conducted at the ENT(Ear, Nose\& throat) Outpatient department (OPD) of a teaching hospital namely Sri Ramachandra Hospital.Patients with different ENT problems attend the OPD among them patients with vertigo also attend the clinic. The period of data collection was from $1^{\text {st }}$ to $30^{\text {th }}$ of June 2013.

\section{Study design}

The Research design adopted for the current study was Randomized control trial.

\section{Population}

Patients with vertigo attending the outpatient department.

\section{Sample}

The study included patients between 20 and 70 years of age of both gender, diagnosed with peripheral vertigo, acute vestibular neuronitis, labrynthitis, benign paroxysmal positional vertigo (BPPV), cooperative and able to perform exercises, able to understand Tamil or English and willing to participate in the study. The exclusion criteria were patients with central vertigo, acute vertigo, M eniere's disease, vascular ischemia, transient ischemic attack, stroke and anxiety.

All patients who fulfilled the inclusion criteria were randomized by lottery method to the assigned groups. 30 lots were prepared by the investigator in which 15 lots were labeled as study group and 15 were labeled as control group. The patients were assigned to the study or control group based on the lots picked by the patients from the box.

\section{Data collection}

The intervention involved vestibular rehabilitation exercises taught and demonstrated by the researcher to the patients on $1^{\text {st }}$ consultation using a booklet for 20 minutes. The exercises included eye, head, sitting, standing and walking exercises. Supervised return demonstration was obtained from the patients. The patients were encouraged to practice the exercises twice daily for 7 days by telephonic reminders. The vertigo patients also received routine care along with the proposed intervention. The patients randomly assigned to the Control group received the routine care. A total of 30 samples both male and female vertigo patients who attended the ENT OPD were selected for the study.

\section{Measurement}

The instruments used for data collection consists of two parts.

\section{Part 1}

A. Demographic variables consist of age, gender, education, occupation, income and marital status.

B. Clinical variables consist of co-morbid conditions, past history of vertigo, medications, measures practiced by self to reduce vertigo and dietary pattern. 
Part 2

Dizziness handicap was assessed with the help of dizziness handicap inventory. It is a standardized tool by G P Jacobson and Neumann C W (1990) with 19 items which are scored as, yes-04, sometimes-02 and no-0. It was graded as mild handicap (16-34), moderate handicap (3652) and severe handicap (54+).

\section{Ethical consideration}

Ethical permission was obtained from the Institutional ethical committee. During the period of data collection the investigator introduced self to the patients and established rapport with them. They were assured that no emotional or physical harm would be done during the course of the study. The intervention was explained to the patients and written informed consent was obtained prior to the initiation of the intervention. The purpose of the study was explained to each subject in the local language and they were told that participation in the study was based on their willingness and it would in no way interfere with their treatment. They were also informed that confidentiality would be maintained and they could withdraw their participation whenever they wanted to do so.

\section{Data analysis}

The SPSS 16 was used for analysis of the data Descriptive statistics such as frequency, percentage, mean and standard deviation and inferential statistics such as paired ' $t$ ' test, independent ' $t$ ' test and Chi square were used for the study. The $p$ value of $\varangle .05$ was considered statistically significant.

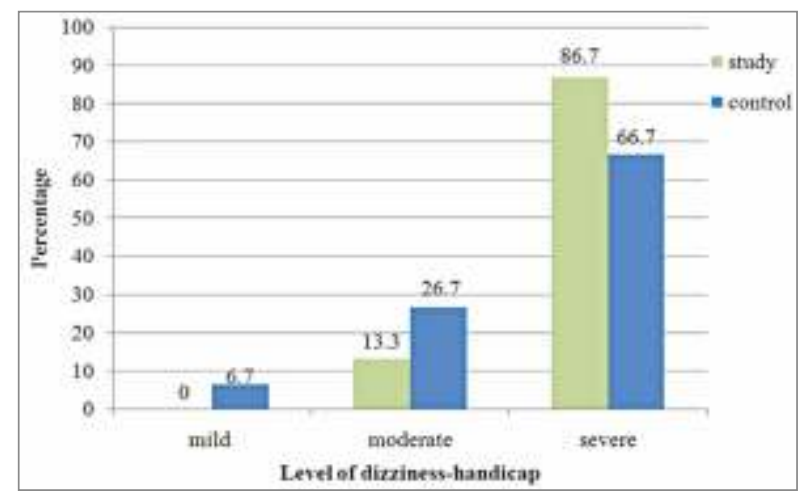

Figure 1 : Percentage distribution of level of dizziness-handicap among the study and the control group during the pretest.

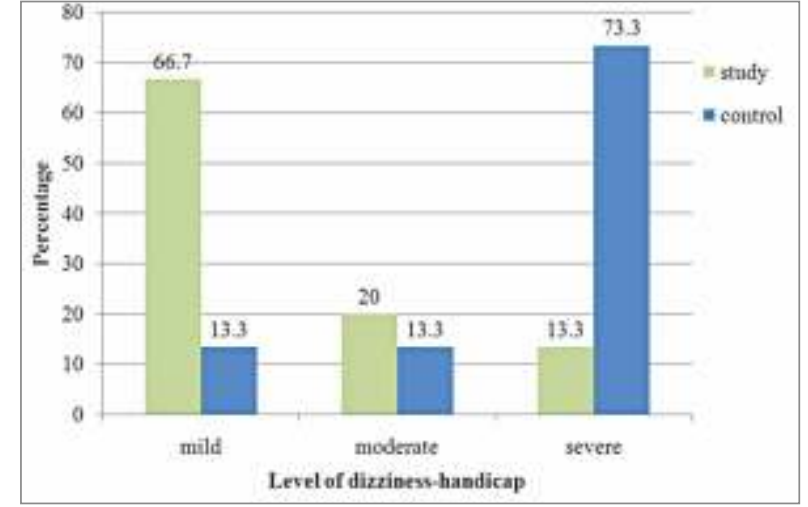

Figure 2 : Percentage distribution of level of dizziness-handicap among the study and the control group during the posttest.

\section{Results}

Patient characteristics:

The patient characteristics taken into account were age, gender, education, occupation, monthly income and marital status.

Table 1 : Frequency and percentage distribution of demographic variables of vertigo patients in the study and the control group

$(\mathrm{N}=30)$

\begin{tabular}{|c|c|c|c|}
\hline Patient characteristics & $\begin{array}{c}\text { Study } \\
\text { group N (\%) }\end{array}$ & $\begin{array}{c}\text { Control } \\
\text { group n (\%) }\end{array}$ & p value \\
\hline Age (in years) & & & \\
\hline a. 20-35 & $2(13.3)$ & $4(26.7)$ & \\
\hline b. 36-50 & $4(26.7)$ & $4(26.7)$ & \\
\hline c. 51-65 & $3(20)$ & $4(26.7)$ & \\
\hline d. 66-80 & $6(40)$ & $3(20)$ & 0.78 \\
\hline Gender & & & \\
\hline a. Male & $7(46.7)$ & $8(53.3)$ & \\
\hline b. Female & $8(53.3)$ & $7(46.7)$ & 0.71 \\
\hline Education & & & \\
\hline a. No formal education & $5(33.3)$ & $8(53.3)$ & \\
\hline b. School & $3(20)$ & $4(26.7)$ & 0.29 \\
\hline c. Diploma/graduate & $7(46.7)$ & $3(20)$ & \\
\hline Occupation & & & \\
\hline a. Unemployed/house wife & $6(40)$ & $6(40)$ & \\
\hline b. Private & $9(60)$ & $6(40)$ & 0.16 \\
\hline c. Government & $0(0)$ & $3(20)$ & \\
\hline Monthly income (in Rs) & & & \\
\hline a. <5000 & $6(40)$ & $3(20)$ & \\
\hline b. 5001-10000 & $6(40)$ & $7(46.7)$ & 0.62 \\
\hline c. 10001-15000 & $2(13.3)$ & $4(26.7)$ & \\
\hline d. >15000 & $1(6.7)$ & $1(6.7)$ & \\
\hline M arital status & & & \\
\hline a. Married & $9(60)$ & $13(86.7)$ & \\
\hline b. Unmarried & $4(26.7)$ & $1(6.7)$ & 0.23 \\
\hline c. Divorced/widowed & $2(13.3)$ & $1(6.7)$ & \\
\hline
\end{tabular}

Table 1 reveals that both the study and control groups were homogenous with regards to their demographic variables 
Table 2 : Frequency and percentage distribution of clinical variables of vertigo patients in the study and the control group

$(\mathrm{N}=30)$

\begin{tabular}{|c|c|c|c|}
\hline $\begin{array}{l}\text { Clinical } \\
\text { variables }\end{array}$ & $\begin{array}{c}\text { Study } \\
\text { group N (\%) }\end{array}$ & $\begin{array}{c}\text { Control } \\
\text { group N (\%) }\end{array}$ & $p$ value \\
\hline \multicolumn{3}{|l|}{ Co morbid conditions } & \multirow{5}{*}{0.938} \\
\hline a. Diabetes & $6(40)$ & $7(46.7)$ & \\
\hline b. Hypertension & $5(33.3)$ & $5(33.3)$ & \\
\hline c. Anemia & $2(13.3)$ & $2(13.3)$ & \\
\hline d. Others & $2(13.3)$ & $1(6.7)$ & \\
\hline Previous history of vertigo & & & \multirow{5}{*}{0.977} \\
\hline a. Nil significant & $6(40)$ & $5(33.3)$ & \\
\hline b. Within the past 6 mon & S. $4(26.7)$ & $5(33.3)$ & \\
\hline c. 6 months to one year & $3(20)$ & $3(20)$ & \\
\hline d. >1 year & $2(13.3)$ & $2(13.3)$ & \\
\hline \multicolumn{3}{|l|}{ Medications } & \multirow[b]{3}{*}{0.143} \\
\hline a. Yes & $5(33.3)$ & $9(60)$ & \\
\hline b. No & $10(66.7)$ & $6(40)$ & \\
\hline \multicolumn{3}{|c|}{ M easures practiced by self to reduce Vertigo } & \multirow[b]{3}{*}{0.456} \\
\hline a. Yes & $10(66.7)$ & $8(53.3)$ & \\
\hline b. No & $5(33.3)$ & $7(46.7)$ & \\
\hline \multicolumn{3}{|l|}{ Dietary pattern } & \multirow{7}{*}{0.464} \\
\hline a. Caffeine intake & & & \\
\hline (i) $<2$ times & $7(46.7)$ & $9(60)$ & \\
\hline (ii) $\geq 2$ times & $8(53.3)$ & $6(40)$ & \\
\hline \multicolumn{3}{|l|}{ b. Alcohol intake } & \\
\hline (i) Once a week & 0 & 0 & \\
\hline (ii) > once a week & 0 & 0 & \\
\hline
\end{tabular}

Table 2 shows that $40 \%$ in the study group and $46.7 \%$ in the control group had diabetes, though majority $40 \%$ in the study group and $33.3 \%$ in the control group did not have any previous history of vertigo majority of the participants had previous history of vertigo ranging from within six months to more than a year. $66.7 \%$ in the study group had taken medicines and $60 \%$ in the control group had not taken medicines for the control of their vertigo $53.3 \%$ in the study group had intake of coffee more than twice a day and $60 \%$ in the control group had coffee intake of less than twice a day. The study participants did not reveal any history of alcohol intake. Though there were differences between the study and control group it was not found to be statistically significant and hence both the groups were homogenous with regards to their clinical variables
Table 3 : Frequency and percentage distribution of level of dizziness-handicap among the vertigo patients in the study and the control group

$(\mathrm{N}=30)$.

\begin{tabular}{|c|c|c|c|c|c|c|}
\hline Duration of & \multicolumn{3}{|c|}{ Study group $(n=15)$} & \multicolumn{3}{|c|}{ Control group ( $n=15)$} \\
\hline the study & & & & & & \\
\hline $\begin{array}{l}\text { Level of } \\
\text { dizziness } \\
\text { handicap }\end{array}$ & $\begin{array}{l}\text { Mild } \\
\text { N (\%) }\end{array}$ & $\begin{array}{c}\text { Moderate } \\
\mathrm{N}(\%)\end{array}$ & $\begin{array}{c}\text { Severe } \\
\mathrm{N}(\%) \mathrm{N}(\%)\end{array}$ & $\begin{array}{c}\text { Mild } \\
\text { N (\%) }\end{array}$ & Moderate & Severe \\
\hline Pretest & - & $2(13.3)$ & $13(86.7)$ & $1(6.7)$ & $4(26.7)$ & $10(66.7)$ \\
\hline Posttest & $10(66.7)$ & $\begin{array}{ll}3 & (20) \\
\end{array}$ & $2(13.3)$ & $2(13.3)$ & $2(13.3)$ & 11 (73.3) \\
\hline
\end{tabular}

Table 3 shows that in the study group, at pretest $86.7 \%$ had severe dizziness handicap whereas in the posttest $66.7 \%$ had mild dizziness handicap. In the control group $66.7 \%$ had severe dizziness handicap in the pretest and 73.3\% had severe dizziness handicap at posttest.

\section{Discussion}

When determining the effectiveness of vestibular rehabilitation on dizziness-handicap there has been a significant change in the level of dizziness-handicap in the study group than that of the control group. During pretest, $2(13.3 \%)$ of them had moderate level of dizzinesshandicap and 13 (86.7\%) had severe level of dizzinesshandicap in the study group. The pretest mean of study group was $66.33 \pm 14.03$ and post-test mean was $31.07 \pm 18.94$. The ' $t$ ' value was -4.336 which was statistically significant at $p=0.0005$. This showed that the study group patients had felt decreased level of dizziness handicap after performance of vestibular rehabilitation exercises. The level of dizziness-handicap among the control group reveals that the pretest mean was $61.33 \pm 15.24$ whereas, post-test mean was $63.87 \pm 22.35$. The calculated ' $\mathrm{t}$ ' value was -0.306 which was not statistically significant. This showed that the patients in the control group did not experience decrease in the level of dizziness-handicap.

A study conducted by M oreira Bittar R S et al (2007) among 52 elderly patients diagnosed with vertigo and other balance disorders were selected and divided into the study and control groups respectively and vestibular rehabilitation exercises were taught to the study group. $84.5 \%$ in the study group and $81.8 \%$ in the control group had reduced vertigo. ${ }^{25}$ 
A study conducted by Prasansuk S et al (2004) on the balance disorders among elderly and the benefit of balance exercises where 60 elderly patients with vertigo were selected and taught vestibular rehabilitation exercises. During follow-up, after 4 weeks it was found that $80 \%$ of the patients had mild dizziness-handicap and $20 \%$ had moderate dizziness-handicap after the patients started to regularly practice vestibular rehabilitation. ${ }^{26}$

This study thus reveals the importance of teaching vestibular exercises to patients and emphasizing the performance of the same regularly which can be done by the nurse who is the first line care giver. It is also imperative that the vestibular rehabilitation component be included in nursing education so that the student nurses will also include this as a component of health education for patients who attend the ENT Outpatient department in

\section{References}

1. Taylor I\& Goodkin HP. Dizziness and vertigo in the adolescent, Otolaryngologic Clinics of North America, 2011; 44 (2), 309-321

2,13.Swartz R \& Longwell P. Treatment of vertigo, American Family Physician, 2005; 71 (6), 1115-1122.

3,11.Neuhauser HK, Von Brevern M , Radtke A, LeziusF, Feldmann M , Ziese T, \& Lempert T. Epidemiology of vestibular vertigo: A neurotologic survey of the general population. Neurology, 2005; 65 (6), 898-904. http://www.ncbi.nlm.nih.gov/pubmed/16186531

4. Schappert SM. Ambulatory care visits to physician offices, hospital outpatient departments, and emergency departments: United States, 1996 Vital and Health Statistics. Series 13, Data from the National Health Survey, 1998; (134), 1-37. http://www.cdc.gov/nchs/data /series/sr_13/sr13_134.pdf

5,20.Sloane $\overline{\mathrm{P}}$, Blazer $\overline{\mathrm{D}}, \&$ George LK. Dizziness in a community elderly population. Journal of the American Geriatrics Society, 1989; 37(4), 101-108.

6. Dros J, M aarsingh OR, Beem L, Van der Horst HE, Ter Riet G, Schellevis FG, \& Van Weert HCPM. Impact of dizziness on everyday life in older primary care patients: a cross-sectional study, Health and Quality of Life Outcomes, 2011:9, 44.

7. Abrol R, Nehru VI, \&Venkatramana Y. Prevalence and etiology of vertigo in adult rural population.Indian Journal of Otolaryngology and Head \& Neck Surgery, 2001; 53 (1), 32-36. http://www.ncbi. nlm.nih.gov/ doi: 10.1007/BF02910976

8. Hain TC. Neurophysiology of vestibular rehabilitation. Neuro Rehabilitation, 2011;29(2), 127-141.

9. Smeltzer SC, Bare BG. HinkleJL and Keever CH. Textbook of medical surgical nursing, Lippincott William \& Wilkins publications, $12^{\text {th }} \mathrm{Ed}$ 2012, 1119-1124.

10. Mira E. Improving the quality of life in patients with vestibular disorders: The role of medical treatments and physical rehabilitation. International Journal of Clinical Practice, 2008; 62 (1), 109-114. http://www.medscape.com/viewarticle/568873 1

12. M endel B, Bergenius J, \& Langius A. Dizziness symptom severity and impact on daily living as perceived by patients suffering from peripheral vestibular disorder.Clinical Otolaryngology and Allied Sciences, 1999; 24 (4), 286-293. doi:10.1046/j.1365-2273.1999. 00269.x

14. Yardley L, Burgneay J, Andersson G, Owen N, Nazareth I\&Luxon L. Feasibility and effectiveness of providing vestibular rehabilitation for dizzy patients in the community.Clinical Otolaryngology and Allied Sciences, 1998; 23 (5), 442-448. http://onlinelibrary.wiley.com/doi/ 10.1046/j.1365-2273.1998.00179.x/pdf order to facilitate a better Quality of life for the patients who have vertigo. As a nurse administrator one can enable availability of pamphlets on vestibular exercises for those patients who do not have access to internet or other media so that they will be able to visualize the exercises and perform them safely at home.

\section{Conclusion}

The study findings support that there is a significant difference in the dizziness-handicap among the patients in the study group who practiced vestibular rehabilitation than those in the control group who did not practice vestibular rehabilitation.

\section{Limitations}

- Some of the exercises were modified according to individual patient needs.

- Telephonic reminders were given to the subjects.

15. Venosa AR \& Bittar RS. Vestibular rehabilitation exercises in acute vertigo. The Laryngoscope, 2007; 117(8), 1482-1487.

16. Agus S, Benecke H, Thum C \& Strupp M. Clinical and demographic features of vertigo: Findings from the REVERT registry. Frontiers in Neurology, 4 MAY, 2013. http://www.ncbi.nlm.nih.gov/pmc/articles /PM C3650561/doi: 10.3389/fneur.2013.00048

17. Yardley L, Barker F, M uller I, Turner D, Kirby S, M ullee M , Little P. Clinical and cost effectiveness of booklet based vestibular rehabilitation for chronic dizziness in primary care: single blind, parallel group pragmatic, randomised controlled trial, BM J, 2012; 54 (2), 694 978.doi: http://dx.doi.org/10.1136/bmj.e2237

18. Lewis SM, Heitkemper MM \& Dirksen SR. Medical surgical nursingassessment and management of clinical problems, Mosby publications, $6^{\text {th }}$ ed, 2004, 1200-1205.

19. Sixt E\& Landahl S. Postural disturbances in a 75-year-old population: I. Prevalence and functional consequences. Age and Ageing, 16, 2013 393-398. http://www.ncbi.nlm.nih.gov/pmc/articles/PM C4498008/ doi: 10.3238/arztebl.2015.0387

21. Ekvall HE \& M agnusson $M$. Vestibular asymmetry predicts falls among elderly patients with multi- sensory dizziness. BM C Geriatrics, 2013; 13(1), 77. http://bmcgeriatr.biomedcentral.com/articles/10.1186/ 1471-2318-13-77 DOl: 10.1186/1471-2318-13-77

22. Kumar RS, Srinivasan I R. Effectiveness of Vestibular Rehabilitation Therapy in Patients Suffering from B.P.P.V.IJSR, June 2013, 2 (6),24-28 http:// www.ijsr.net/archive/v2i6/IJSRON20131081.pdf

23. Han BI, Song HS, \& Kim JS. Vestibular rehabilitation therapy: Review of indications, mechanisms, and key exercises. Journal of Clinical Neurology (Korea), 2011;78 (6),998-1008. http://www.ncbi.nlm.nih. gov/pmc/articles/PM C3259492/ 10.3988/jcn.2011.7.4.184

24. HanssonEE, Månsson NO\&Håkansson A. Balance performance and self-perceived handicap among dizzy patients in primary health care. Scandinavian Journal of Primary Health Care, 23, 2013, 2215-220. http:// www.tandfonline.com/ doi/full/10.1080/02813430500287299 DOI:10.1080/02813430500287299

25. Bittar RSM \& Barros C, de G C. Vestibular rehabilitation with biofeedback in patients with central imbalance. Brazilian Journal of Otorhinolaryngology, 2011; 77(3), 356-361. http://dx.doi.org /10.1590/S1808-86942011000300014

26. Prasansuk S, Siriyananda C, Nakorn AN, Atipas S \& Chongvisal S Balance disorders in the elderly and the benefit of balance exercise. Journal of the Medical Association of Thailand, 2004; 87(10), 1225-1233. https://www researchgate net/publication/8166052 